\title{
Eco-friendly, Salt-Free Reactive Dyeing by Cationization of Cotton with Amino Acids Obtained from Soya Bean Hull
}

\section{Dessiea $A^{1}$ and Govindanb $\mathbf{N}^{2 *}$}

${ }^{1}$ Kombolcha Institute of Technology, Wollo University, Kombolcha, Ethiopia

${ }^{2}$ Textile Chemistry Research and Innovation Centre, Ethiopian Institute of Textile and Fashion Technology, Bahir Dar University, Bahir Dar, Ethiopia

\begin{abstract}
Cotton acquires negative charge in aqueous medium and thus repels negatively charged dye anion during dyeing. Such repulsion between fibre and dye is offset by using large quantity of salt in dye bath, particularly for reactive dyes. A low dye bath exhaustion also leads to low dye fixation of reactive dyes on cotton. Thus, the use of high salt concentration and low reactive dye fixation lead to environmental problems related to highly coloured effluent with high salt content. Hence, modifying the cotton fibre to increase dye-fibre interaction is therefore the best route to overcome the lack of affinity for cotton to reactive dyes making salt-free reactive dyeing. However, majority of the chemicals used for imparting cationic sites in cotton are themselves not eco-friendly. This paper presents the possibility of salt-free reactive dyeing of cotton by modifying the surface of cotton fabric using soya bean hull. The cationization of cotton was carried out with natural amino acid extract obtained by acid hydrolysis from soya bean hull and cationized in the pad-dry-curing process to investigate its dyeability with reactive dye in both a conventional alkaline dye bath and salt-free acidic dye bath. FTIR spectroscopy confirmed that there was a change in chemical composition after cationization by pad-dry-cure technique. The color strength of modified cotton was better than that of conventional sample which is 3.9865 and 3.7135 respectively. The extent of improvement was $4 \%$ in dye exhaustion, $3.34 \%$ in dye fixation and $7.12 \%$ in the total dye utilization was achieved in this study. The wash, dry rubbing and perspiration fastness of the cationized cotton was found almost similar to that of the normal dyeing. There was a slight increase in flexural rigidity and crease recovery angle in the cationized cotton. The tensile strength of cationized and the conventional dyed cotton were found almost the same. The cationized cotton dyeing technique reduced the BOD, COD and TDS by around $32.4 \%, 27.3 \%$ and $75.4 \%$ respectively. It was revealed that soya bean hull was found to be effective for cationization in salt-free dyeing of cotton.
\end{abstract}

Keywords: Cotton; Cationization; Extracted amino acid; Salt-free dyeing; Reactive dyes; Color strength; Fastness

\section{Introduction}

The most popular dyes for dyeing cotton are the reactive dyes because of their brightness of shade, wide colour gamut, flexible application procedures and all-round good colour fastness properties of the resultant dyeing $[1,2]$. In recent years, reactive dyes maintain the largest annual consumption in the world among the dyes used for cotton dyeing. But some problems, such as low dye utilization, large amount of electrolyte used and high volume of wastewater discharged, always exist in the application of reactive dyes [3]. With growing popularity of reactive dyes for dyeing of cotton, environmental problems associated with their use have received attention [4]. Inadequate dye bath exhaustion and dye fixation pose the problem of color effluents. Therefore, a dyeing procedure leading to high dye fixation could be of great benefit to minimize the environmental problems [5-11]. Modifying the cotton fibre to increase dye-fibre interaction is therefore the best route to overcome the lack of affinity for cotton to reactive dyes making salt-free or low-salt reactive dyeing to become an important research subject in recent years $[12,13]$.

Researchers have investigated different kinds of cationic reagents for the cationization of cotton. Both chemical and physical modifications of cotton with compounds including monomers and polymers were employed. Epoxy compounds, chlorotriazine type quaternary compounds, $\mathrm{N}$ methylolacrylamide, choline chlorides etc. are the monomers commonly used in modification of cotton fiber. Synthetic polymers, polyepi-chloro-hydrin dimethylamine, polyamide epichlorohydrin type polymers, poly-(4-vinylpyridine) quaternary ammonium compounds and dendritic polymers, and biopolymerschitosan, starch and their derivatives are typical compounds used in modification of cotton [14]. Chemical modification of cotton has been earlier studied by many researchers in different ways and means, which are widely available in the literature. Majority of the chemicals used for introducing cationic sites in cotton are themselves not safe environmentally. However, these problems can also be overcome by improving the dye substantively of cotton in the absence of salt or with low salt additions. One such approach is cationization of cotton which is critically explored in this investigation. Therefore, there is a need to explore the potentiality of the cationization route using eco-friendly chemicals. The use of natural amino acid, a polymer derived from waste soya bean, is a step forward in this investigation.

As an important root to obtaining the desired dyeing performance with existing dyes, chemical modification of cotton fiber to impart cationic charges have been widely researched in recent years. By introducing cationic groups into cotton fibers, the affinity of anionic dyes for cotton was significantly improved, which allows the dyeing of cotton fabrics without salt and to improve reactive dye utilization. With less chemical, water, and energy consumption, the dyeing of cationized cotton is a potentially environmentally responsible process $[5,6,11]$.

*Corresponding author: Govindanb N, Professor, Textile Chemistry Research and Innovation Centre, Ethiopian Institute of Textile and Fashion Technology, Bahir Dar University, Bahir Dar, Ethiopia, Tel: +251-98-462-4403; E-mail: gnalankilli@yahoo.com

Received November 11, 2018; Accepted December 14, 2018; Published December 24, 2018

Citation: Dessiea A, Govindanb N (2018) Eco-friendly, Salt-Free Reactive Dyeing by Cationization of Cotton with Amino Acids Obtained from Soya Bean Hull. J Textile Sci Eng 8: 385. doi: 10.4172/2165-8064.1000385

Copyright: @ 2018 Dessiea A, et al. This is an open-access article distributed under the terms of the Creative Commons Attribution License, which permits unrestricted use, distribution, and reproduction in any medium, provided the original author and source are credited. 
The amounts of salt required for exhaustion and the depth of shade for heavy reactive dye formulations lead to large amounts of salt released in the effluent and discharged to streams and rivers. Salt concentrations may be as high as $100 \mathrm{~g} / \mathrm{L}$ for dark shades. Salt may be considered as one of the most toxic chemicals emitted from textile mills because of the quantity released, albeit not necessarily the toxicity of the salt itself. Finally, if the effluent must be treated, i.e. desalinated, the additional cost of this processing step makes the desalination unattractive just from an economical point of view $[12,15]$.

Cationization of cotton is one of the most widely researched modifications in recent years since both direct and reactive dyes carry anionic charges and they exhibit high affinity for positively charged cotton. Numerous chemicals and methods have been used to introduce cationic groups into cotton fiber $[13,16]$. By introduction of cationic groups into cotton fibers, the affinity of reactive dyes for cotton can be significantly improved. The ionic attractions between cationized cotton and reactive dyes can result in increased dye uptake, reduced or no electrolyte use, less dye washing off and less water and energy consumption. The environmental problems caused by dye and salt in effluent can be potentially mitigated by cationization pretreatment of cotton.

Based on the molecular weight; the cationic reagents can be divided into two groups, monomeric reagents and polymeric reagents. Epoxy compounds, chlorotriazine type quaternary compounds, $\mathrm{N}$ methylolacrylamide, choline chloride etc. are the monomers commonly used in modification of cotton fiber [14]. Moreover, most of the chemicals are non-biodegradable as well as non-renewable [12]. Most of the techniques developed so far for reducing salt concentration in cotton dyeing are complicated in nature. Some of these methods are successful only for highly reactive dyes. Under this background, efforts were made to find out easier method for salt- free reactive dyeing of cotton. Since most synthetic chemicals used for cationization of cotton are not safe environmentally, waste soya bean, the natural waste product, will be used as a substitute for improving cotton dyeability in this investigation

Soybean hulls are a by-product of the extraction of oil from soybean seeds. After entering the oil mill, soybeans are screened to remove broken and damaged beans, and foreign material. The beans are then cracked, and their hulls, which mainly consist of the outer coats, are removed. Hulls are fibrous materials with no place in human food [17]. It was observed that amino acid content in this hydrolysed soya bean waste contains a total of 17 types of amino acids, out of which glutamic acid and arginine contents are the highest [18]. In this investigation, an attempt was conducted to modify cotton fabrics by pretreatment with natural amino acid extracted from soya bean hull. In addition, salt-free reactive dyeing of cationized cotton was carried out with the consideration of environment and cost of dyeing.

\section{Materials and Methods}

$100 \%$ half bleached plain-weave cotton fabric was used in this study with $21 \mathrm{Ne}, 24$ ends per inch, 18 picks per inch and having an average areal density of $145 \mathrm{~g} / \mathrm{m}^{2}$ has been used in the present work. The chemicals required for extraction, cationization and dyeing are; hydrochloric acid, sodium hydroxide, sodium chloride, sodium carbonate, acetic acid, citric acid, wetting agent, levelling agent and standard detergent. Reactive red HE3B (C.I. Reactive Red 120)) was used throughout the study. In this investigation, the following equipment/apparatus and machineries were used for extraction, cationization and dyeing. The major equipment used in these studies were Mesdan Padder Lab 300 for padding in cationization, S.D. Mini Dryer for drying and curing during cationization and after dyeing, Infrared Laboratory dyeing machine used for dyeing, Digital Tearing Strength Tester, S.D.L Stiffness Tester, Mesdan Crease Angle Recovery Tester 3101, Perkin Elmer FT-IR Spectrometer, Auto-Wash for washing fastness, Crock-Meter for rubbing fastness, Perspirometer for perspiration fastness, PerkinElmer UV/VIS Spectrometer Lambda 25 for measuring colour absorption, Gretag Macbeth Color Eye 3100 reflectance spectrophotometer for measuring K/S, BOD Incubator, COD Digestion Apparatus, TDS Meter, Digital pH Meter.

Minitab 18 software with Taguchi method was used for determination of the optimum number of experiment. SPSS statistical tool was used to analyse statistical results. For optimization of extraction parameters a four factor four level Taguchi design of experiment was used. A total of sixteen experiments (Tables 1 and 2) were carried out as per the design of experiment software. The experiment was conducted at room temperature. For optimization of cationization a five factor five level Taguchi design of experiment was used. A total of twenty five experiments were carried out as per the design experiment software. The speed (contact time) of the machine was maintained constant throughout the experiment and percentage pick-up was also calculated.

Soya bean was first purchased from local market and then dried by exposing it to sunlight. The dried soya bean was cracked in mill to remove the hull from the seed. Then the soya bean hulls were ground using laboratory cutting mill. The ground soya bean hulls were dissolved at different concentration of hydrochloric acid for different treatment time at room temperature in order to determine the optimum extraction parameters. The $\mathrm{pH}$ of the solution was brought to

\begin{tabular}{|c|c|c|c|}
\hline Exp. No. & Concentration $\%$ & Combinations of Time in Hrs & Combinations of MLR \\
\hline $1,2,3,4$ & 2.5 & $8,16,24,32$ \\
\hline $5,6,7,8$ & 5 & $8,16,24,32$ \\
\hline $9,10,11,12$ & 7.5 & $8,16,24,32$ \\
\hline $13,14,15,16$ & 10 & $8,16,24,32$ \\
\hline
\end{tabular}

Table 1: Taguchi design for optimization of extraction parameters.

\begin{tabular}{|c|c|c|c|c|c|}
\hline Exp. No. & Pressure in Bar & $\begin{array}{c}\text { Combinations of Drying } \\
\text { Temp. }{ }^{\circ} \mathrm{C}\end{array}$ & $\begin{array}{c}\text { Combinations of Drying } \\
\text { Time in Min. }\end{array}$ & $\begin{array}{c}\text { Combinations of Curing } \\
\text { Temp. }{ }^{\circ} \mathrm{C}\end{array}$ & $\begin{array}{c}\text { Combinations of Curing } \\
\text { Time in Min. }\end{array}$ \\
\hline $1,2,3,4,5$ & 1 & $90,95,100,105,110$ & $1,2,3,4,5$ & $110,120,130,140,150$ & $1,2,3,4,5$ \\
\hline $6,7,8,9,10$ & 2 & $90,95,100,105,110$ & $2,3,4,5,1$ & $130,140,150,110,120$ & $4,5,1,2,3$ \\
\hline $11,12,13,14,15$ & 3 & $90,95,100,105,110$ & $3,4,5,1,2$ & $150,110,120,130,140$ & $2,3,4,5,1$ \\
\hline $16,17,18,19,20$ & 4 & $90,95,100,105,110$ & $4,5,1,2,3$ & $120,130,140,150,110$ & $5,1,2,3,4$ \\
\hline $21,22,23,24,25$ & 5 & $90,95,100,105,110$ & $5,1,2,3,4$ & $140,150,110,120,130$ & $3,4,5,1,2$ \\
\hline
\end{tabular}

Table 2: Taguchi design for optimization of cationization conditions. 
$5.5-6.0$ by addition of dilute sodium hydroxide solution. The solution is then filtered to remove any solid and the filtrate was taken as soya bean hull extracted amino acid solution.

Extraction was tried at $2.5 \%, 5 \%, 7.5 \%$ and $10 \%$ concentration of hydrochloric acid; 1:5, 1:10, 1:15 and 1:20 material to liquor ratio; 8,16 , 24 and 32 hours of treatment time for at room temperature. Extraction parameters were then optimized depending upon their efficiency. Percentage dissolution (efficiency) was calculated using equation (1) by drying the residue after treatment.

$$
\text { Efficiency }(\%)=\frac{\left[\mathrm{W}_{1}-\mathrm{W}_{2}\right]}{\mathrm{W}_{1}} \times 100
$$

Where $\mathrm{W}_{1}$ is the original weight of soya powder and $\mathrm{W}_{2}$ is the weight of the residual soya.

The application of soya extract containing amino acid solution on bleached cotton was done by padding technique in a laboratory twobowl padding mangle (two dips and two nips) at $100 \%$ wet pickup. The squeezing of the treated fabric was tried at 1,2,3,4, and 5 bar pressure of the squeezing rollers. The padded and squeezed cotton fabrics were dried at a temperature of $90,95100,105$ and $110^{\circ} \mathrm{C}$ for $1,2,3,4$ and 5 minutes. Subsequently curing was tried at $110,120,130,140$ and 150 ${ }^{\circ} \mathrm{C}$ for $1,2,3,4$ and 5 minutes of curing time. Cationization parameters were optimized based on minimum scorching of fabric, dyeing evenness and maximum K/S values. Cationization was confirmed using FT-IR by checking presence of additional functional groups, measuring the dye uptake phenomenon using UV/VIS Spectrometer and the K/S value using Colour Eye 3100 .

The untreated and cationized fabric was dyed in laboratory infrared dyeing machine with a material-to-liquor ratio of 1:20 using Reactive red HE3B (C.I. Reactive red-120) for $2 \%$ shade following the standard procedure. The dye bath did not contain salt during the application of reactive dye unless otherwise specified. The color strength was measured using color eye 3100 reflectance spectrophotometer result in $\mathrm{L} \mathrm{x}$ a x b, D-65 and $10^{\circ}$ observer, the color intensities $(\mathrm{K} / \mathrm{S})$ of the cationized samples at different conditions was determined. The reflectance $(\mathrm{R})$ value of dyed fabric at the maximum wavelength of absorbency $(\lambda \max )$ was found and the K/S was calculated using the built-in software of the computer color matching system. Kubelka-Munk equation (2) given below has been used for K/S determination.

$$
\frac{\mathrm{K}}{\mathrm{S}}=\frac{(1-\mathrm{R})^{2}}{2 \mathrm{R}}
$$

Where, $\mathrm{K}$ is the light absorption coefficient, $\mathrm{S}$ is the light scattering coefficient while $\mathrm{R}$ is the $\mathrm{D} 65 / 10$ light reflection. K/S values were calculated based on reflection values of untreated and treated cotton fabrics. This value symbolizes the reduction ratio of light owing to absorption and scattering achieved based on reflectance. The absorbance (optical density) of the dye solution was measured before and after the dyeing processes using a Lambda 25 PerkinElmer UV/VIS Spectrometer at the maximum wavelength of absorbance $(\lambda \max )$. The percentage of dye bath exhaustion (E) was calculated using equation (3):

$$
\% \mathrm{E}=\frac{\left(\mathrm{A}_{0}-\mathrm{A} 1\right)}{\mathrm{A}_{0}}
$$

Where $A_{0}$ is the absorbance of dye solution before the commencement of dyeing and $A_{1}$ is the absorbance of dye solution after the dyeing process. The reflectance $(\mathrm{R})$ values at all wavelengths were measured. The maximum K/S value of dyed fabric at the certain wavelength was measured by using Colour Eye 1500 before and after soaping. The percentage of dye fixation was calculated using equation (4).

$$
\% \mathrm{~F}=\frac{(\mathrm{K} / \mathrm{S})_{\mathrm{a}}}{(\mathrm{K} / \mathrm{S})_{\mathrm{b}}} \times 100
$$

Where $(\mathrm{K} / \mathrm{S}) \mathrm{b}$ and $(\mathrm{K} / \mathrm{S}) \mathrm{a}$ are the colour strength before soaping and after soaping respectively. The total dye utilization percentage (\%T) was calculated by using equation (5).

$$
\% \mathrm{~T}=\frac{\mathrm{E} \times \mathrm{F}}{100}
$$

ISO 105 C06 2002 method for wash fastness. ISO 105 F09 2009 standard for Rubbing fastness ISO 105 B02 standard for Light fastness, ISO 105F10 standard for Perspiration fastness, ASTM D1388-2007 standard for Stiffness of fabric, ASTM D1423-83 standard for tear strength, ASTM D5035-95 standard Tensile strength, IS: 4681-1968 standard for Crease recovery, ASTM D629-99 for FTIR measurements for studying the available functional groups, IS:3025-part 44: 1993 standard for Biological Oxygen Demand (BOD), IS:3025-part 58: 2006 standard for Chemical Oxygen Demand (COD), IS:3025-part 16: 1984 standard for Total Dissolved Solid (TDS) were used.

\section{Result and Discussions}

\section{Optimization of extraction parameters}

The extraction of natural amino acid from soya bean hull powder was carried out at different extraction conditions. For extraction of natural mixed amino acid solution from soya bean hull powder the extraction parameters which were optimized includes concentration of $\mathrm{HCl}$, treatment time and material to liquor ratio. The extraction was done at room temperature.

Table 3 showed that the efficiency of extraction of amino acid was maximum (92.6\%) at experiment number 15. Therefore, the optimized extraction conditions were $10 \% \mathrm{HCl}, 24$ hours treatment time and 1:10 material to liquor ratio. The extraction was carried out at room temperature $\left(27 \pm 3^{\circ} \mathrm{C}\right)$. This means that the treatment of the soya powder with hydrochloric acid at this optimized extraction conditions gives maximum efficiency of extraction of amino acid solution. In addition to this, the extraction of amino acid above $10 \%$ concentration of hydrochloric acid is expensive.

Figure 1 indicates that higher percentage yield (dissolution) was obtained when soya bean hull powder was treated with $10 \%$ hydrochloric acid for 24 hours at 1:10 material to liquor ratio. This treatment was performed at room temperature. It can be concluded that as the concentration of hydrochloric acid increases, the percentage dissolution also increases. However, the use of hydrochloric acid above $10 \%$ in order to get higher yield is not cost effective and the increment is also not that much higher as compared to the optimized extraction conditions.

\section{Optimization of cationization parameters}

Bleached cotton fabric was cationized with the extracted mixture of aqueous amino acid solution using a two-bowl laboratory padding mangle at $100 \%$ wet pick-up. The padding of the sample was tried at different squeezing pressure to optimize the cationization conditions. 


\begin{tabular}{|c|c|c|c|c|c|c|}
\hline No. of Experiment & Concentration of $\mathrm{HCl}(\%)$ & Time (hr) & MLR & Original Weight (g) & Residual Weight (g) & Efficiency (\%) \\
\hline 1 & 2.5 & 8 & $1: 5$ & 20 & 9.19 & 54.05 \\
\hline 2 & 2.5 & 16 & $1: 10$ & 20 & 8.53 & 57.35 \\
\hline 3 & 2.5 & 24 & $1: 15$ & 20 & 7.23 & 63.85 \\
\hline 4 & 2.5 & 32 & $1: 20$ & 20 & 6.47 & 67.65 \\
\hline 5 & 5 & 8 & $1: 10$ & 20 & 5.64 & 71.8 \\
\hline 6 & 5 & 16 & $1: 15$ & 20 & 5.21 & 73.95 \\
\hline 7 & 5 & 24 & $1: 20$ & 20 & 4.96 & 75.2 \\
\hline 8 & 5 & 32 & $1: 5$ & 20 & 5.10 & 74.5 \\
\hline 9 & 7.5 & 8 & $1: 15$ & 20 & 4.65 & 76.75 \\
\hline 10 & 7.5 & 16 & $1: 20$ & 20 & 3.97 & 80.15 \\
\hline 11 & 7.5 & 24 & $1: 5$ & 20 & 4.14 & 79.3 \\
\hline 12 & 7.5 & 32 & $1: 10$ & 20 & 3.47 & 82.65 \\
\hline 13 & 10 & 8 & $1: 20$ & 20 & 3.16 & 84.2 \\
\hline 14 & 10 & 16 & $1: 5$ & 20 & 2.95 & 85.25 \\
\hline 15 & 10 & 24 & $1: 10$ & 20 & 1.48 & 92.6 \\
\hline 16 & 10 & 32 & $1: 15$ & 20 & 1.94 & 90.35 \\
\hline
\end{tabular}

Table 3: Optimization of extraction conditions

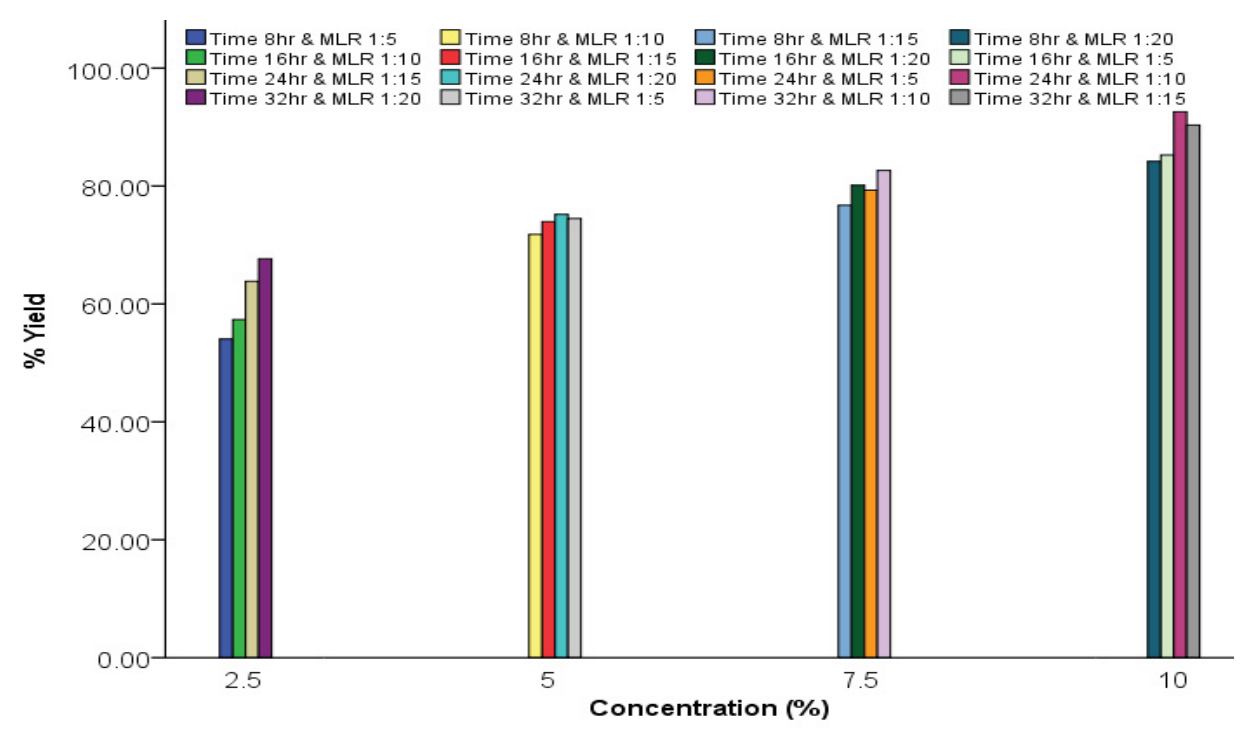

Figure 1: Optimization of extraction conditions.

The treated fabric was then subjected to drying and then curing at different drying temperature, drying time, curing temperature and curing time to optimize the cationization of cotton as shown in Table 2.

\section{Confirmation of cationization: Infrared analysis}

Both the untreated control cotton fabric and the cationized cotton samples were examined in a FTIR spectrophotometer. The presence of soya extract amino acid in the cationized cotton fabric was confirmed with the use of FTIR technique.

The result of the above Figure 2 showed that there was a change in chemical composition after cationizing by pad-dry-cure method; the amino acid was fixed to the fabric. The broader absorption band between 3000 and $3600 \mathrm{~cm}^{-1}$ is attributed to $\mathrm{OH}$ stretching vibration forming hydrogen bonds in the cellulose molecule. This common absorption band is composed of two small vibrations located at 3296 $\mathrm{cm}^{-1}$ (attributed to intermolecular hydrogen bonds) and $3341 \mathrm{~cm}^{-1}$ (attributed to intra-molecular hydrogen bonds). The peaks observed at $2895-2908 \mathrm{~cm}^{-1}$, which is attributed to $-\mathrm{CH}_{2}$ asymmetric vibrations.
Other common absorption bands appearing in different intensities in the spectra are $1424 \mathrm{~cm}^{-1}$ which is the characteristics of $-\mathrm{CH}_{2}-$ symmetrical bending.

One new notable absorption bands appearing in spectrum (b) in strong to medium intensities at $1648 \mathrm{~cm}^{-1}$ clearly depicts the presence of quaternary nitrogen group $(-\mathrm{N}+-3 \mathrm{R})$ in which the ' $\mathrm{R}$ ' groups are substituted with amino groups. The other new absorption band was observed at peak $2345 \mathrm{~cm}^{-1}$ which is the characteristics of $-\mathrm{CH}_{2}-$ stretching. Such absorption bands, however, are practically nonexistent in the spectrum of bleached untreated cotton (a). Apart from this, FTIR spectra (b) for soya extracted amino acid treated cotton shows a larger intensity, which is probably due to additional formation of ester-COO- linkage. The treatment of cotton with amino acid of soya in the presence of heat and acidic catalyst forms an ester linkage incorporating the free $-\mathrm{NH}_{2}$ group in modified cotton, which in presence of acid can form $-\mathrm{NH}^{+3}$ in the cationized cotton. Such spectral analysis reveals that the amino groups bind with the primary alcohol group of cellulose with the help of ester linkage between them. 


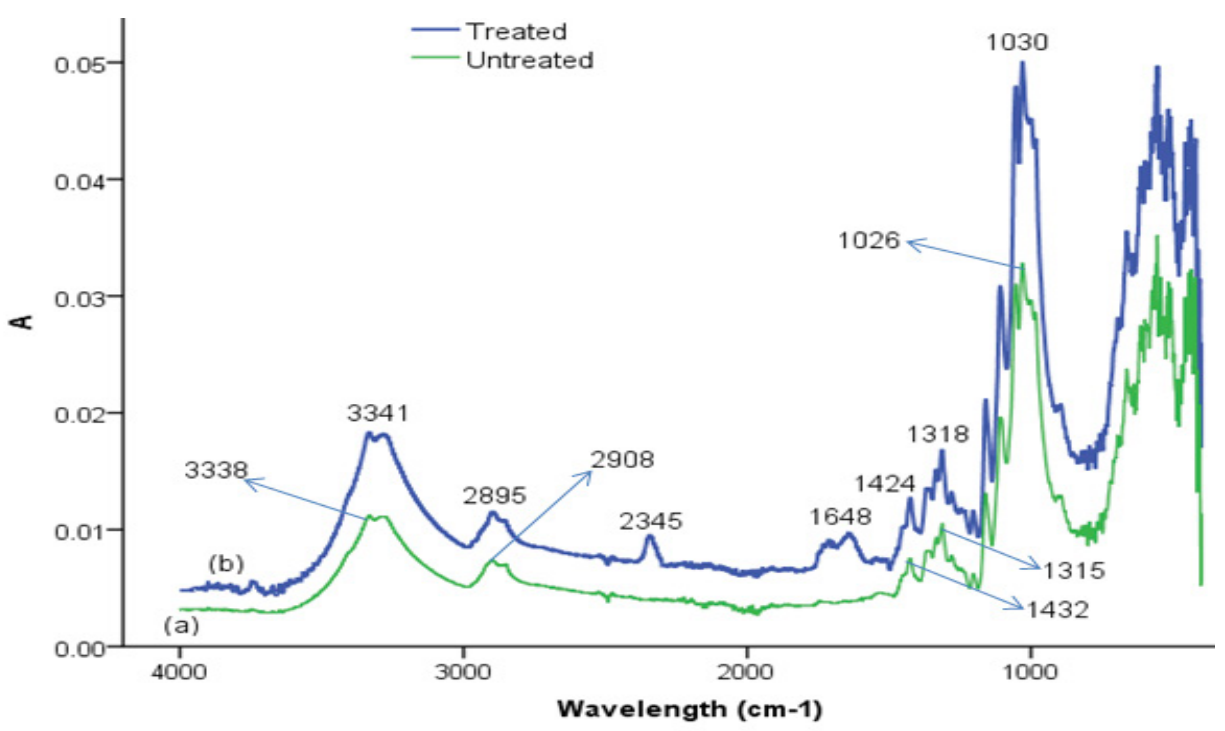

Figure 2: Infrared spectra of (a) untreated and (b) treated bleached cotton.

\section{Dyeing}

The color intensities $(\mathrm{K} / \mathrm{S})$ is used to express the reduction ratio of light owning to absorption and scattering achieved based on reflectance. The higher the color intensities is the more dye is fixed on the fabric and the low dye variation effect will have on the fabric because light is reflected more in a uniform surface.

The application of soya extract amino acid solution on cotton was carried out using pad-dry-cure technique. All the above sample fabric was cationized using pad-dry-cure technique. Prior treatment of cotton with soya extract following a pad-dry-cure technique increases the fixation of reactive dyes. Dyeing of cationized and conventional cotton with Reactive red HE3B (C.I. Reactive Red 120) was performed as per the standard method prescribed by the dye manufacturers.

From the above Table 4 and Figure 3, it can be seen that sample which was squeezed at 1 bar pressure and then dried at $100^{\circ} \mathrm{C}$ for 3 min and subsequently cured at $130^{\circ} \mathrm{C}$ for $3 \mathrm{~min}$ gave a high $\mathrm{K} / \mathrm{S}$ value therefore it was used for comparison with conventionally dyed fabric. This highest K/S value (3.9865) was achieved at the highest add-on percentage (40.24\%). In addition to this, the fabric which was cationized at this optimized cationization conditions shows minimum scorching of the fabric. Therefore, it was concluded that the optimized cationization conditions of cotton fabric with amino acid solution of soya are squeezing of the impregnated cotton at 1 bar pressure and then drying the padded fabric at $100^{\circ} \mathrm{C}$ for $3 \mathrm{~min}$ and subsequently curing at $130^{\circ} \mathrm{C}$ for $3 \mathrm{~min}$. This gives higher color strength value as compared to other samples. At this cationization conditions, a higher percentage add-on was also obtained. The K/S values of dyed fabric with HEreactive red dye with different dyeing techniques but similar conditions are shown in Table 5. It shows that a higher K/S values was obtained with cationized cotton fabric.

The increased in K/S value in the fabric treated with soya extract amino acid solution point towards the existence of higher amount of the dye absorbed in the treated fabric. It is also confirmed that the colour yield was improved in the cationized fabric than the untreated cotton as given in Figure 4.
Figure 4 shows that the color strength of the cationized cotton fabric was higher than the untreated one. The cationized cotton fabric which was dyed with reactive dye via salt-free acid bath dyeing process has given higher K/S values than the conventionally dyed fabric with salt. The improved dye uptake of treated cotton fabric may be attributed to the generation of positive charge.

In the non-conventional reactive dyeing process with acid bath at the start of dyeing and alkaline bath after addition of alkali for fixation, there is strong attraction between the cationic dye sites on the modified cotton and the anionic dyes existed which led to obtain a very high exhaustion rates without addition of electrolytes to the dye-bath. In addition to this, the improvement of dye-uptake in the cationized (chemically modified) cotton was due to creation of supplementary hydroxyl groups which increases dye attachment sites. This proves the success of soya extract amino acid solution treatment in enabling cotton fabric to be dyed in absence of salt. In this Reactive red HE3B (C.I. Reactive Red 120), for a higher potential difference between reactive dye anion and the amino group of soya-modified cotton, there is more reactive dye attracted towards the cationic group of amino acid residue fixed to cotton by cationization with soya extract.

From Table 6 it was observed that there was a no significant difference in the ' $\mathrm{L}^{*}$ ' value (lightness) between the treated and untreated fabric. Also, between the cationized and uncationized fabric there was no significant difference in the trichromacity co-ordinates. The cationized cotton showed lower ' $a$ ' value, meaning redder; while the uncationized cotton showed higher ' $b$ ' value, meaning bluer. The cationized fabric showed lower yellowness index as compared to the conventional fabric. The whiteness index was also lower which shows that the cationized cotton have higher depth of shade. This confirms that high amount of dyes are absorbed by the cationized fabric.

\section{Effect of cationization on dye exhaustion and fixation}

Effect on percentage exhaustion (\% E): The optical density of dye solution before and after the dyeing was measured using UV/ VIS spectrophotometer at the maximum wavelength of absorbance $\left(\lambda_{\max }\right)$. The maximum absorbance at $520 \mathrm{~nm}$ wavelength was taken to determine the dye exhaustion percentage. 
Citation: Dessiea A, Govindanb N (2018) Eco-friendly, Salt-Free Reactive Dyeing by Cationization of Cotton with Amino Acids Obtained from Soya Bean Hull. J Textile Sci Eng 8: 385. doi: 10.4172/2165-8064.1000385

Page 6 of 9

\begin{tabular}{|c|c|c|c|c|c|c|c|}
\hline No. & Squeezing Pressure (bar) & Drying temp. ( $\left.{ }^{\circ} \mathrm{C}\right)$ & Drying time (min) & Curing Temp. $\left({ }^{\circ} \mathrm{C}\right)$ & Curing time (min) & Add-on \% & $\mathrm{K} / \mathrm{S}$ value \\
\hline 1 & 1 & 90 & 1 & 110 & 1 & 36.3 & 2.7051 \\
\hline 2 & 1 & 95 & 2 & 120 & 2 & 38.71 & 3.0158 \\
\hline 3 & 1 & 100 & 3 & 130 & 3 & 40.24 & 3.9865 \\
\hline 4 & 1 & 105 & 4 & 140 & 4 & 38.56 & 3.4091 \\
\hline 5 & 1 & 110 & 5 & 150 & 5 & 35.33 & 3.1495 \\
\hline 6 & 2 & 90 & 2 & 130 & 4 & 37.96 & 3.7921 \\
\hline 7 & 2 & 95 & 3 & 140 & 5 & 37.66 & 3.4165 \\
\hline 8 & 2 & 100 & 4 & 150 & 1 & 34.22 & 3.7006 \\
\hline 9 & 2 & 105 & 5 & 110 & 2 & 32.68 & 3.1827 \\
\hline 10 & 2 & 110 & 1 & 120 & 3 & 38.56 & 3.8279 \\
\hline 11 & 3 & 90 & 3 & 150 & 2 & 35.88 & 3.5801 \\
\hline 12 & 3 & 95 & 4 & 110 & 3 & 33.3 & 3.1693 \\
\hline 13 & 3 & 100 & 5 & 120 & 4 & 32.34 & 3.4851 \\
\hline 14 & 3 & 105 & 1 & 130 & 5 & 37.62 & 3.4544 \\
\hline 15 & 3 & 110 & 2 & 140 & 1 & 36.84 & 3.6251 \\
\hline 16 & 4 & 90 & 4 & 120 & 5 & 30.28 & 3.1594 \\
\hline 17 & 4 & 95 & 5 & 130 & 1 & 29.86 & 3.3320 \\
\hline 18 & 4 & 100 & 1 & 140 & 2 & 33.88 & 3.2130 \\
\hline 19 & 4 & 105 & 2 & 150 & 3 & 32.77 & 3.2029 \\
\hline 20 & 4 & 110 & 3 & 110 & 4 & 31.36 & 2.8176 \\
\hline 21 & 5 & 90 & 5 & 140 & 3 & 29.25 & 3.0375 \\
\hline 22 & 5 & 95 & 1 & 150 & 4 & 32.83 & 2.9199 \\
\hline 23 & 5 & 100 & 2 & 110 & 5 & 32.69 & 3.1007 \\
\hline 24 & 5 & 105 & 3 & 120 & 1 & 31.02 & 2.8482 \\
\hline 25 & 5 & 110 & 4 & 130 & 2 & 30.62 & 3.5680 \\
\hline
\end{tabular}

Table 4: Optimization of cationization conditions and their $\mathrm{K} / \mathrm{S}$ values.

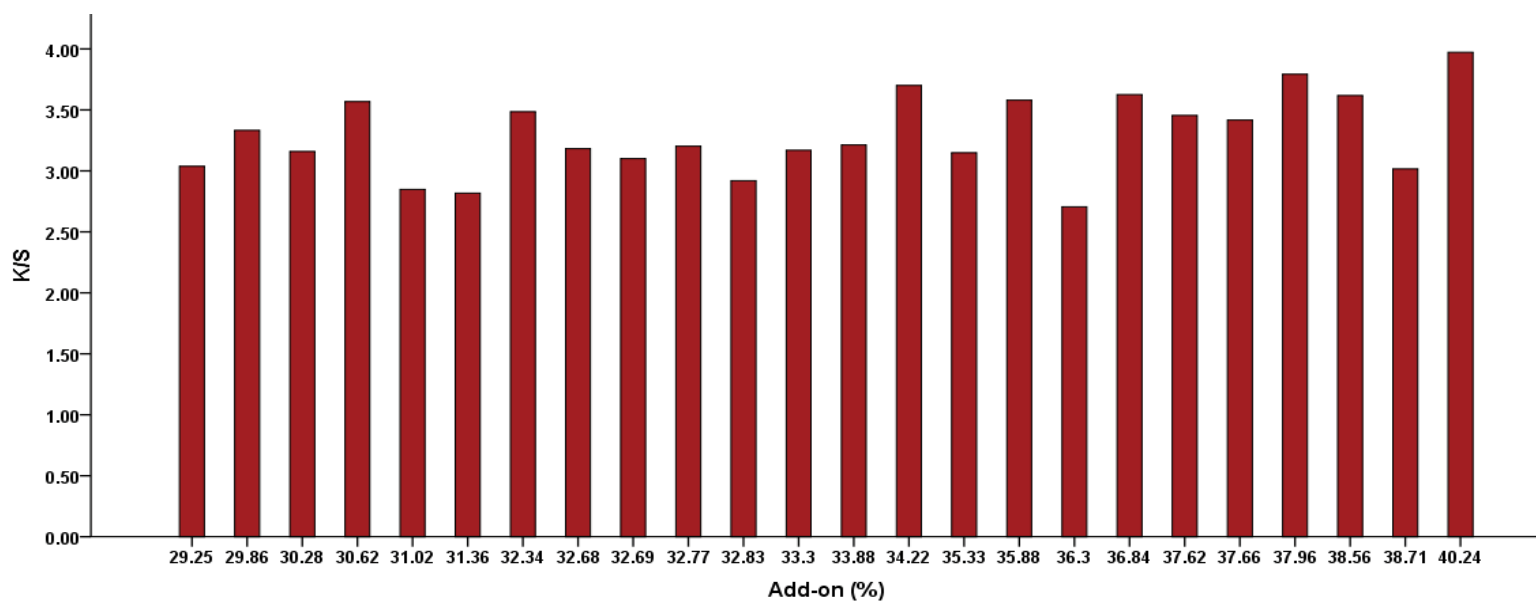

Figure 3: Optimization of cationization parameters

\begin{tabular}{|c|c|c|}
\hline Samples & Reflectance (\%) & K/S \\
\hline Untreated and salt free & 14.99 & 2.4105 \\
\hline Conventional & 10.73 & 3.7135 \\
\hline Cationized & 10.31 & 3.9865 \\
\hline
\end{tabular}

Table 5: K/S value of different samples at $540 \mathrm{~nm}$ wavelength.

The result of the Table 7 showed that the cationized fabric had high dye exhaustion percentage indicating that there was better utilization of dyes. This also shows that the effluent that leaves the dye bath was less colored. The improvement of the dye exhaustion was by $60 \%$ from the untreated and salt free dyed sample and by $3.91 \%$ from the conventional sample. The improved dye uptake of the modified materials may be attributed to the generation of positive charge. The dye-fibre attraction in this reactive dye and amino acid-modified system in the absence of salt is expected to depend chiefly on the ionic attraction between negatively charged

reactive dyes and electron-deficient protonated amino groups of amino acid duly bound to cotton via ester linkage. Above exhaustion pattern of reactive dyes for amino acid-modified cotton is the consequence of such force of attraction between protonated pendant prior grafted amino groups of cotton and negatively charged reactive dyes that ultimately led to improved levels of dye transfer into the amino acid-cationized cotton substrates from an aqueous dye bath.

Effect on percentage fixation (\% F): The result of the Table 8 showed that the cationized cotton provided higher fixation percentage 


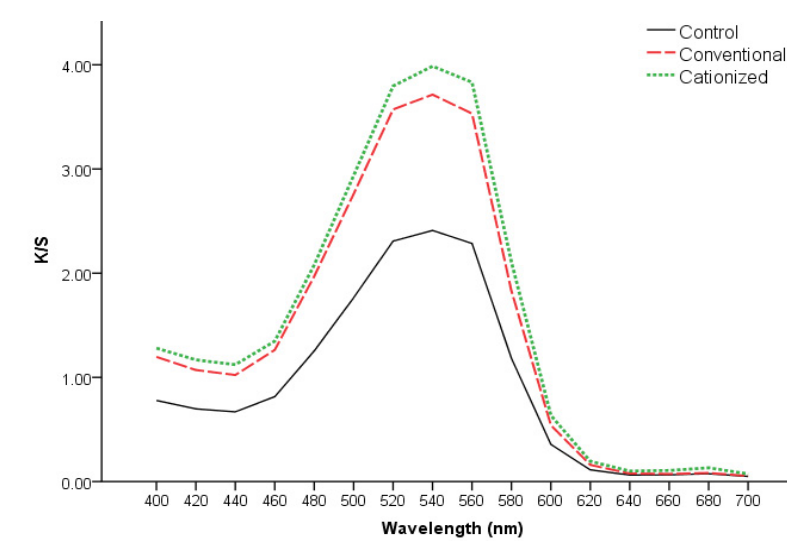

Figure 4: K/S values of cationized and untreated cotton after dyeing.

\begin{tabular}{|c|c|c|c|c|}
\hline $\begin{array}{c}\text { Color } \\
\text { Factors }\end{array}$ & $\begin{array}{c}\text { Half Bleached } \\
\text { cotton }\end{array}$ & $\begin{array}{c}\text { Untreated and } \\
\text { Salt- free }\end{array}$ & Conventional & Cationized \\
\hline $\mathrm{L}^{*}$ & 88.45 & 57.67 & 52.98 & 52.51 \\
\hline $\mathrm{a}^{*}$ & -0.09 & 40.49 & 43.69 & 40.99 \\
\hline $\mathrm{b}^{*}$ & 7.02 & -6.47 & -5.20 & -6.37 \\
\hline $\mathrm{X}$ & 69.31 & 34.87 & 30.14 & 28.90 \\
\hline $\mathrm{Y}$ & 73.01 & 25.61 & 21.63 & 20.90 \\
\hline $\mathrm{Z}$ & 69.70 & 31.98 & 25.72 & 25.94 \\
\hline $\mathrm{X}$ & 0.3269 & 0.3772 & 0.3968 & 0.3940 \\
\hline $\mathrm{Y}$ & 0.3443 & 0.2770 & 0.2723 & 0.2743 \\
\hline Yellowness & 14.32 & 34.56 & 47.02 & 38.80 \\
\hline Whiteness & 39.12 & 66.06 & 55.02 & 53.13 \\
\hline
\end{tabular}

Table 6: Color eye 3100 reflectance spectrophotometer result in $L \times a \times b, D-65$ and $10^{\circ}$ observer.

\begin{tabular}{|c|c|c|c|}
\hline Samples & $\begin{array}{c}\text { Maximum } \\
\text { Absorbance } \\
\text { before dyeing }\left(A_{0}\right)\end{array}$ & $\begin{array}{c}\text { Maximum } \\
\text { Absorbance } \\
\text { after dyeing }\left(A_{1}\right)\end{array}$ & $\begin{array}{c}\text { Percentage } \\
\text { Exhaustion } \\
(\% \mathrm{E})\end{array}$ \\
\hline $\begin{array}{l}\text { Untreated and salt } \\
\text { free }\end{array}$ & 5.7935 & 3.7217 & 35.76 \\
\hline Conventional & 5.7935 & 0.8065 & 86.08 \\
\hline Cationized & 5.7935 & 0.6037 & 89.58 \\
\hline
\end{tabular}

Table 7: Percentage exhaustion of cationized cotton.

\begin{tabular}{|c|c|c|c|}
\hline Sample & $\begin{array}{c}\text { Maximum K/S } \\
\text { before soaping at } \\
\mathbf{5 4 0} \mathbf{~ n m}\end{array}$ & $\begin{array}{c}\text { Maximum K/S } \\
\text { after soaping at } \\
\mathbf{5 4 0} \mathbf{~ n m}\end{array}$ & $\begin{array}{c}\text { Fixation } \\
\text { percentage } \\
\mathbf{( F} \text { \%) }\end{array}$ \\
\hline $\begin{array}{c}\text { Untreated and } \\
\text { Salt free }\end{array}$ & 3.8973 & 2.4105 & 61.85 \\
\hline Conventional & 4.2032 & 3.7135 & 88.35 \\
\hline Cationized & 4.3616 & 3.9865 & 91.40 \\
\hline
\end{tabular}

Table 8: Percentage fixation of cationized cotton.

than the uncationized cotton dyed with salt. The improvement of fixation percentage was around $32 \%$ from the untreated salt free dyed cotton and by around 3.34\% from the uncationized cotton fabric dyed with salt. The reason behind the improvement in the dye fixation was because there is less chance for hydrolysis of dyes. The improvement in fixation was also reflected in the washing fastness ratings shown in Table 8. Alkali addition, during fixation, encourages the reaction of the dye with the fibre or with the water present in the system. The rate of the reaction depends on the reactivity of the dye. The dyes exhausted by the fibre preferentially react with the fibre, therefore higher exhaustion results in higher degree of fixation also. The fixation of dyes was also found to be improved greatly on the cationized cotton. Such treatment was, therefore, adequate in screening the negative surface charge of the fibre and thus enabling appreciable exhaustion and fixation of reactive dye even in the absence of salt.

Higher incorporation of amino acid into cotton via esterification evidently caused higher extent of fixation of reactive dyes in consequent to higher initial transfer of reactive dyes. The fixation of reactive dyes appears to be dependent on the initial absorption of reactive dyes on soya extract amino acid treated cotton.

Effect on percentage dye utilization (\%T): The Table 9 shows that the cationized cotton provided higher dye utilization percentage than the conventional sample fabric dyed with salt. The dye utilization was improved from 76.05 to $81.88 \%$ by cationization of cotton with amino acid extracted from soya. The extent of improvement of dye utilization was by around $72.98 \%$ from the untreated and salt free dyed fabric and by around $7.12 \%$ from the conventional method. From this it can be concluded that, the dyeing of cationized cotton can save approximately $7.12 \%$ dye.

Effect of cationization on colour fastness: Color fastness to washing of the cationized fabric was compared with conventionally dyed cotton fabric. It was assessed in respect of color change and staining as shown in Table 10

Table 10 shows that the wash fastness of the cationized cotton fabric was almost similar to the conventional dyed cotton fabric and there was no staining in all cases. Both dyeing methods scored very good grade by assessment with the standard grey scale reading. This may be due to the formation of strong ionic bond between the fibre and the dye because it is equally good as the covalent bond that normally links the dye and fibre. In addition to this, it also confirms the effectiveness of dye fixation due to pretreatment with soya extract amino acid solution for salt free dyeing. A modified dyeing process employed for soya extract amino acid solution cationized cotton having a starting acidic dye bath $\mathrm{pH}$ and a final alkaline fixation $\mathrm{pH}$ appears to have no significant

\begin{tabular}{|c|c|}
\hline Sample & Percentage total dye utilization (\%T) \\
\hline Untreated and Salt free & 22.12 \\
\hline Conventional & 76.05 \\
\hline Cationized & 81.88 \\
\hline
\end{tabular}

Table 9: Percentage dye utilization of cationized cotton.

\begin{tabular}{|c|c|c|c|}
\hline Fastness/ Treatment & $\begin{array}{c}\text { Untreated \& salt- } \\
\text { free }\end{array}$ & Conventional & Cationized \\
\hline Wash fastness & & & \\
\hline Change in colour & $4 / 5$ & $4 / 5$ & $4 / 5$ \\
\hline Staining on white & 5 & $4 / 5$ & $4 / 5$ \\
\hline Dry Rubbing fastness & & & \\
\hline Change in colour & $4 / 5$ & $4 / 5$ & $4 / 5$ \\
\hline Staining on white & $4 / 5$ & $4 / 5$ & $4 / 5$ \\
\hline Wet Rubbing fastness & & & 4 \\
\hline Change in colour & 4 & $4 / 5$ & 4 \\
\hline Staining on white & 4 & $4 / 5$ & \\
\hline Alakaline perspiration fastness & & & $4 / 5$ \\
\hline Change in colour & $4 / 5$ & $4 / 5$ & $4 / 5$ \\
\hline Staining on white & $4 / 5$ & $4 / 5$ & \\
\hline Acidic perspiration fastness & & & $4 / 5$ \\
\hline Change in colour & $4 / 5$ & $4 / 5$ & $4 / 5$ \\
\hline Staining on white & $4 / 5$ & $4 / 5$ & \\
\hline Light fastness & & & \\
\hline Change in colour & 6 & 6 & \\
\hline
\end{tabular}

Table 9: Percentage dye utilization of cationized cotton. 


\begin{tabular}{|c|c|c|c|}
\hline Fastness/ Treatment & Untreated \& salt-free & Conventional & Cationized \\
\hline Wash fastness & & & \\
\hline Change in colour & $4 / 5$ & $4 / 5$ & $4 / 5$ \\
\hline Staining on white & 5 & $4 / 5$ & $4 / 5$ \\
\hline $\begin{array}{c}\text { Dry Rubbing fastness } \\
\text { Change in colour }\end{array}$ & $4 / 5$ & $4 / 5$ & $4 / 5$ \\
\hline $\begin{array}{c}\text { Staining on white } \\
\text { Wet Rubbing fastness }\end{array}$ & $4 / 5$ & $4 / 5$ & $4 / 5$ \\
\hline Change in colour & 4 & $4 / 5$ & 4 \\
\hline $\begin{array}{c}\text { Staining on white } \\
\text { Alakaline perspiration } \\
\text { fastness }\end{array}$ & 4 & $4 / 5$ & 4 \\
\hline $\begin{array}{c}\text { Change in colour } \\
\text { Staining on white }\end{array}$ & $4 / 5$ & & \\
\hline Acidic perspiration fastness & & $4 / 5$ & $4 / 5$ \\
\hline $\begin{array}{c}\text { Change in colour } \\
\text { Staining on white }\end{array}$ & $4 / 5$ & $4 / 5$ & $4 / 5$ \\
\hline Light fastness & $4 / 5$ & $4 / 5$ & $4 / 5$ \\
\hline Change in colour & 6 & & \\
\hline \multicolumn{2}{|c|}{ Table 10: Fastness properties. } \\
\hline
\end{tabular}

adverse effect on the fixation process of reactive dye for natural amino acid cationized cotton.

Color fastness to rubbing is a basic test used to determine the quality of a colored fabric. This test is designed to determine the amount of colour transferred from the surface of coloured textile materials to other surface by rubbing. Color fastness to rubbing of the cationized fabric was compared with conventionally dyed cotton fabrics. The result shows that the dry rubbing fastness of all samples was giving not any change and all samples scored very good grade. This may be because of the formation of strong ionic bond between the anionic dye molecule and the cationic cotton surface. The cationized sample also scored good grade in the wet rubbing fastness but one step down than that of the conventional dyed sample. This may be due to the tendency of the hydrolysed dyes being able to form hydrogen bond with amino acid of soya on cotton surface. Color fastness to perspiration of the cationized fabric was compared with conventionally dyed cotton fabric. The results show that cationized (treated) fabric had similar color change with conventionally dyed cotton. The staining properties of both the unmodified and cationized fabric is also almost similar in both acidic and alkaline media. For dyed uncationized and cationized samples with HE- reactive dye fastness properties shows very good perspiration fastness. No staining in all cases was also observed in the polyester fabric. Light fastness measures the resistance to color change of dyed textile when exposed to day light. The light fastness of both untreated fabric dyed with salt and the cationized sample dyed salt free was compared. The fastness was assessed by comparing the change in color of the specimen with that of the standards. The result showed that the light fastness of the untreated fabric was excellent and equivalent with the conventional fabric. The light fastness of fabric generally above 4 is acceptable. From the above results, Cationized cotton dyed sample resulted one step downed light fastness compared to other samples. The presence of an aliphatic molecule between the dye and fiber may be disturbing the stable electronic configuration of dye that leads to the shifting of electrons to the higher energy state and subsequent disintegration of dye by the photons of light rays. The reason for one step reduction in the light fastness of cationized cotton was most likely due to the fact that the dye penetrability was good in the cationized fibers with the intended dye fixation method, which assisted in obtaining high light fastness.
Effect of cationization on physical properties: In addition to dyeing performance and colourfastness, commercial viability is also dependent on physical properties of the treated or cationized samples. All the fabric samples were conditioned for 48 hours at $65 \%( \pm 5 \%)$ relative humidity and $27^{\circ} \mathrm{C}\left( \pm 2^{\circ} \mathrm{C}\right)$ temperature before carrying out any physical testing. The results of physical properties are shown in Table 11 . The tensile strength of conventionally dyed fabric and the cationized samples were found to be almost same. The result shows that a small loss $(0.92 \%)$ in tensile strength was observed. This loss of strength might be due to thermal degradation of cotton during curing. The tear strength that means the tendency of the fabric to start, continue or propagate an initial cut of the conventional and the cationized fabric has no such significant reduction. This might be due to the less tendency of the reduction in the number of intermolecular hydrogen bonds by cross-linking of molecule by the cationic reactants. As a result, the cationization using amino acid doesn't significantly affect the tear strength of cotton. Basically, the hypothesis which says cationization occurs mainly at methyl hydroxyl groups of cotton cellulose i.e. at carbon number six (C6) and without breaking the intermolecular hydrogen bonds was very well proved by the fact that the strength is not affected due to modification with amino acid.

It is evident that the flexural rigidity of conventional dyed and cationized cotton does not reflect any major variation. The cationization of cotton with amino acid slightly increased the flexural rigidity of cotton as a result of pretreatment. The increase in flexural rigidity shows that fabric became slightly stiffer as a result of cationization of cotton with amino acid extract of soya. In addition, the stiffness of a fabric in bending is very dependent on its thickness, the thicker the fabric, the stiffer it is if all other factors remain the same. There was an increase in crease recovery angle of the fabric when treated with amino acid solution. The increase in the crease recovery angle as a result of pretreatment may be explained as follows. There might be crosslinking of amino acid of soya between the cellulosic molecules. These cross-links hinder the molecular and fibrillar slippage and stabilise the structure, thereby increasing the crease recovery angle.

Effect of cationization on effluent load: The results of measured BOD, COD and TDS is shown in Table 12. The result indicates that cationized cotton dyeing technique reduced the BOD by around $32.4 \%$, the COD by around $27.3 \%$ and the TDS by around $75.4 \%$ from the conventional methods. This effluent analysis results clearly depict that the process with amino acid treatment and salt free dyeing shows an effluent load lower than the conventional method. This result not only shows that there is a minimization of the amount of effluent discharged to the environment but also a reduction in the effluent treatment cost.

The cationized cotton dyeing approach produces lesser effluent loads than that of the conventional dyeing. This is because of the absence of salt addition in the dye bath. The wastewater effluent consists of less effluent load as the maximum fixation of dye is assured through cationization. The other important point obtained from the cationic dyeing process was the low volume of water requirement during the wash-off process. This is due to the less quantity of unfixed and hydrolysed dyes. Hence, the dyes deposited on the surface of the fabric can be easily removed through one step soaping in the modified cotton. So, a significant savings in process costs was achieved from the cationized cotton dyeing approach. The most important part of the cationization technique is the reduction of TDS in the effluent as this cannot be removed from the effluent easily and needs capital intensive and costly treatments. 


\begin{tabular}{|c|c|c|c|c|c|c|}
\hline \multirow{2}{*}{ Properties/ Test direction } & \multicolumn{3}{|c|}{ Conventional } & \multicolumn{3}{|c|}{ Cationized cotton } \\
\hline & Weft way & Warp way & Overall & Weft way & Warp way & Overall \\
\hline Tensile strength (N) & 444 & 528 & 486 & 439 & 524 & 481.5 \\
\hline Tear strength (N) & 21.85 & 23.01 & 22.43 & 21.81 & 22.86 & 22.335 \\
\hline Bending Length (cm) & 2.00 & 2.22 & 2.11 & 2.02 & 2.25 & 2.135 \\
\hline Flexural Rigidity (mg-cm) & 14.5 & 19.83 & 17.03 & 14.94 & 11.39 & 17.64 \\
\hline Crease Recovery Angle (i) & 94 & 102 & 196 & 96 & 108 & 204 \\
\hline
\end{tabular}

Table 11: Effect of cationization on Physical properties.

\begin{tabular}{|c|c|c|}
\hline Samples & Conventional & Cationized \\
\hline BOD $(\mathbf{m g} / \mathbf{L})$ & 1,42 & 96 \\
\hline COD $(\mathbf{m g} / \mathbf{L})$ & 1,692 & 1,230 \\
\hline TDS $(\mathbf{p p m})$ & 1,469 & 3,62 \\
\hline
\end{tabular}

Table 12: Effect of cationization on effluent load.

\section{Conclusion}

The present investigation provided an alternative method of salt free dyeing of cotton fabric with reactive dyes by modifying cotton with soya bean hull amino acid by acid hydrolysis. A comparison of reactive dyed soya-modified cotton and reactive dyed unmodified cotton with respect to their dyeing performance, fastness properties and physical property has been made. Pad-dry-cure method has been used to apply the extracted amino acid solution on cotton. The infrared analysis after cationization of cotton confirmed that there was a change in chemical composition; the amino acid was fixed to the fabric.

Cotton treated with amino acid extracted from soya bean hull provided cationic dye sites and thus has been dyed with Reactive Red HE3B (C.I. Reactive Red 120) without electrolyte, giving excellent results. The results on the different reactive groups indicated that the type of reactive groups of reactive dyes had no influence on the dyeing properties of the cationized cotton fabrics. Dyeing of the cationized cotton with slightly acidic dye bath at the start of dyeing and alkaline dye bath after the addition of alkali gave better color strength than the comparable dyeing on uncationized cotton by the conventional method. The extent of improvement was $4 \%$ in dye exhaustion, 3.34\% in dye fixation and $7.12 \%$ in the total dye utilization was achieved from this investigation. The dye hydrolysis is reduced in cationized dyeing as the dye exhaustion percentage of cationized dyeing is higher than conventional dyeing. In addition to this, both the dyeing time and temperature were reduced by 15 and 10 minutes respectively in the cationized cotton dyeing technique. Compared with untreated cotton dyed with a conventional dyeing procedure, the cationized cotton fabrics dyed without electrolytes have adequate and quite comparable colourfastness to laundering, dry crocking and perspiration but slightly reduced wet crocking fastness. The flexural rigidity and crease recovery angle was slightly increased in the cationized cotton. The tensile strength of conventionally dyed fabric and the cationized cotton were found to be almost same. Apart from this, the cationized cotton dyeing technique resulted in a reduction of $32.4 \%$ in BOD, $27.3 \%$ in COD and $75.4 \%$ in TDS as compared to the conventional methods. Moreover, the cationic dyeing process greatly reduced, the amount of water usage and time required to adequately rinse and remove hydrolysed reactive dye. So, a significant savings in process costs was also achieved from the cationized cotton dyeing method. Therefore, from dyeing, economic and environmental point of view, it was concluded that using this natural material for cationization is a good substitute for synthetic cationizing agents. This provides both a strategy for reducing risks and pollutant from salt and unutilized dye and also creates an opportunity for new markets and new businesses that could be implemented for selling of the waste product of soya bean in soya oil processing industries.

Based on all achieved results of this work, it was revealed that cotton fabric after cationization can be dyed with environmentally friendly and most popular fibre reactive dyes without any use of salt during its dyeing and thus can cut down the dye effluent load. Cationization treatment of cotton for improved dyeability thus provides an enormous scope for further investigation to suit the today's need of greener technology.

\section{References}

1. Lewis DM, Vo LT (2007) Dyeing cotton with reactive dyes under neutral conditions. Coloration Technology 123: 306-311.

2. Ristić N, Ristić I (2012) Cationic Modification of Cotton Fabrics and Reactive Dyeing Characteristics. J Eng Fabr Fib 7: 113-121.

3. Ma W, Jz Y (2017) Development of functional polymers in modification of cotton for improving dyeability of reactive dyes. The Proceedings of the 3rd international conference on functional molecules, (2002) Citeseer.

4. Periyasamy AP, Dhurai B, Thangamani K (2011) Salt-free dyeing-a new method of dyeing on lyocell/cotton blended fabrics with reactive dyes. Autex Research J 11: 14-17

5. Chattopadhyay D (2001) Cationization of cotton for low-salt or salt-free dyeing Ind J Fib Text Res 26: 108-115.

6. Chattopadhyay D, Chavan R, Sharma J (2007) Salt-free reactive dyeing of cotton. Int J Cloth Sci Technol 19: 99-108.

7. Montazer M, Malek R, Rahimi A (2007) Salt-free reactive dyeing of cationized cotton. Fib Poly 8: 608-612.

8. Allegre C, Moulin P, Maisseu M, Charbit F (2006) Treatment and reuse of reactive dyeing effluents. J Memb Sci 26: 15-34.

9. Ramasamy M, Kandasaamy $P$ (2005) Effect of cationization of cotton on it's dyeability. Ind J Fib Text Res 30: 315-323.

10. Kannan MSS, Gobalakrishnan M, Kumaravel S, Nithyanadan R, Rajashankar $\mathrm{K}$, et al. (2006) Influence of cationization of cotton on reactive dyeing. Text Appa Technol Manag 5: 23-30.

11. Fu S (2016) Studies on dyeing cationized cotton, North Carolina State University.

12. Farrell MJ (2017) Cationic Cotton Prepared with Hydrophobic Alkyl Chlorohydrin Quats: A New Fiber with New Properties, Sustainable Cotton Dyeing, AATCC International Conference 2017, Charlotte, N.C., USA

13. Shahidi S, Wiener J, Ghoranneviss M (2013) Surface modification methods for improving the dyeability of textile fabrics. Eco-Friendly Textile Dyeing and Finishing. InTech.

14. Aktek T, Millat AM (2017) Salt Free Dyeing of Cotton Fiber-A Critical Review. Intern J Text Sci 6: 21-33.

15. Fu S, Hinks D, Hauser P, Ankeny M (2013) High efficiency ultra-deep dyeing of cotton via mercerization and cationization. Cellulose 20: 3101-3110.

16. Bhuiyan MR, Shaid A, Khan M (2014) Cationization of cotton fiber by chitosan and its dyeing with reactive dye without salt. Chem Mat Engin 2: 96-100

17. Ipharraguerre I, Clark J (2003) Soyhulls as an alternative feed for lactating dairy cows: a review. J Dairy Sci 86: 1052-1073.

18. Samanta AK, Mukhopadhyay A, Konar A, Shome D, Kar TR (2016) Dyeability of chemically modified cotton muslin fabric using natural amino acid extract from soya bean seed. J Text Res 86: 2179-2192. 